\title{
Regional variations in the extent and pattern of grey matter demyelination in multiple sclerosis: a comparison between the cerebral cortex, cerebellar cortex, deep grey matter nuclei and the spinal cord
}

\author{
C P Gilmore, ${ }^{1}$ I Donaldson, ${ }^{1}$ L Bö, ${ }^{2,3}$ T Owens, ${ }^{4}$ J Lowe, ${ }^{5}$ N Evangelou'
}

${ }^{1}$ Department of Neurology, Queens Medical Centre NHS Trust, Nottingham, UK; ${ }^{2}$ Department of Neuropathology, VU Medical Centre, Amsterdam, The Netherlands; ${ }^{3}$ National Competence Centre for MS, Department of Neurology, Haukeland University Hospital, Bergen, Norway: ${ }^{4}$ Department of Economics, University of Nottingham, UK; ${ }^{5}$ Department of Neuropathology, University of Nottingham, UK

\section{Correspondence to:}

Dr C P Gilmore, Department of Neurology, B Floor Medical

School, Queen's Medical Centre

NHS Trust, Nottingham NG7

2UH, UK; chris.gilmore@

nottingham.ac.uk

Received 8 March 2008 Revised 7 May 2008 Accepted 12 May 2008 Published Online First 1 October 2008

\begin{abstract}
Background: Substantial grey matter (GM) demyelination occurs in both the cerebral cortex and spinal cord in multiple sclerosis (MS). GM demyelination also occurs in the cerebellar cortex and the deep GM nuclei of the brain. However, no study has made a direct "within subject" comparison of the extent of GM pathology between these regions.

Aim: To examine the extent and pattern of GM demyelination in the motor cortex, cingulate gyrus, cerebellum, thalamus and spinal cord in MS.
\end{abstract}

Methods: Postmortem study using material from 14 MS cases and three controls. Sections were taken from the five predetermined areas and stained for proteolipid protein. The extent of GM and white matter (WM) demyelination was assessed in each region.

Results and conclusion: Overall, $28.8 \%$ of the GM was demyelinated compared with $15.6 \%$ of the WM $(p<0.001)$, with demyelination being greater in the GM than in the WM at each of the anatomical sites. There was substantial variation in the extent of demyelination between the different CNS regions. GM demyelination was most extensive in the spinal cord and cerebellum while WM demyelination was most prominent in the spinal cord.

The factors influencing plaque topography in multiple sclerosis (MS) are not fully understood. The majority of white matter (WM) plaques occur in a perivenular distribution, with areas containing a high density of small veins and venules-such as the periventricular WM, leucocortical junction and the WM tracts of the brainstem and spinal cordshowing a preponderance for demyelination. ${ }^{1}$ While it has been recognised that MS also involves grey matter (GM) structures, the conventional myelin stains used in these studies grossly underestimate the true extent of this GM pathology. ${ }^{2-4}$

In comparison, myelin protein immunohistochemistry is more sensitive for detecting GM demyelination, demonstrating extensive demyelination in the cerebral cortex and spinal cord GM in MS. ${ }^{4-6}$ However, it is unclear whether GM demyelination is more prominent in the spinal cord, the cerebral cortex or other GM structures; comparisons between studies are likely to be confounded by differences in MS subtype, disease duration, age, gender, etc. Therefore, in this study, we used autopsy material to quantify GM and WM demyelination in the cerebral cortex, cerebellum, spinal cord and thalamus, allowing us to make "within subject" comparisons of the extent of demyelination in these regions.

\section{MATERIALS AND METHODS \\ Clinical material}

Formalin fixed, paraffin embedded autopsy material was obtained from 14 pathologically confirmed MS cases and three controls (MS Brain Bank, London, UK). Patients with MS (one man, 13 women) were aged 44-81 years (mean 56.6, median 56) with disease durations of 6-32 years (mean 23.7, median 22). Eleven cases had secondary progressive MS (SPMS), two primary progressive MS (PPMS) and one relapsing remitting MS (RRMS). The controls (two women aged 69 and 78 years, one man aged 35 years) had no clinical or pathological evidence of neurological disease. The local research ethics committee approved the study.

\section{Preparation of the sections}

Sections $(5 \mu \mathrm{m})$ were taken from seven predetermined areas of the CNS, irrespective of the macroscopic appearance: the motor cortex, cingulate gyrus, cerebellum, thalamus and spinal cord (transverse sections from the cervical, thoracic and lumbar levels). Sections were stained for proteolipid protein (PLP) as described previously. ${ }^{6}$

\section{Measurement of GM and WM lesions}

The PLP stained sections were scanned in a slide scanner to produce a digital image. Using these images the GM/WM boundary and the MS lesions were manually outlined (AnalySIS Pro running SIS software; Olympus UK). The control cases were used to establish normal regional variations in myelin staining. Lesions were defined as sharply demarcated areas, characterised by either complete myelin loss or markedly reduced myelin density (likely to represent partially remyelinated lesions). The proportion of GM that was demyelinated $\left(\mathrm{P}_{\mathrm{GMd}}\right.$, ie, the area of demyelinated $\mathrm{GM}$ as a percentage of the total GM cross sectional area) and the proportion of WM that was demyelinated ( $\left.\mathrm{P}_{\mathrm{WMd}}\right)$ was then calculated.

\section{Statistical analysis}

Paired $t$ tests were used to compare $\mathrm{P}_{\mathrm{GMd}}$ with $\mathrm{P}_{\mathrm{WMd}}$ in each anatomical region (ie, each tissue section contained both GM and WM, hence paired tests). Correlations between $\mathrm{P}_{\mathrm{GMd}}$ and $\mathrm{P}_{\mathrm{WMd}}$ were 
Table 1 Frequency of lesions in the five anatomical regions

\begin{tabular}{|c|c|c|c|c|c|}
\hline \multirow[b]{2}{*}{ Location } & \multirow[b]{2}{*}{$\begin{array}{l}\text { Tissue sections } \\
\text { examined }\end{array}$} & \multicolumn{2}{|c|}{ Areas of GM demyelination } & \multicolumn{2}{|c|}{ Areas of WM demyelination } \\
\hline & & No & $\begin{array}{l}\text { Proportion that are } \\
\text { pure GM lesions }(\%)^{*}\end{array}$ & No & $\begin{array}{l}\text { Proportion that are } \\
\text { pure WM lesions }(\%)\end{array}$ \\
\hline Cingulate & 13 & 20 & 90 & 9 & 77.8 \\
\hline Motor cortex & 12 & 28 & 100 & 2 & 100 \\
\hline Deep GM & 13 & 36 & 87.5 & 13 & 61.5 \\
\hline Cerebellum & 13 & 79 & 50.6 & 41 & 56.1 \\
\hline Spinal cord & 37 & 42 & 16.7 & 70 & 14.3 \\
\hline
\end{tabular}

Spinal cord values represent the pooled results from the three cord levels.

*Pure GM lesions (ie, lesions that are restricted to the GM and do not impinge on the adjacent WM).

GM, grey matter; WM, white matter.

Figure 1 PLP immunostaining of the cerebral cortex in controls $(A, C)$ and in patients with multiple sclerosis $(B, D-F)$. $(A, C)$ In controls, PLP staining is reduced in $\mathrm{GM}$ in comparison with WM, particularly in the superficial cortex (C). (B) Subpial lesion extending over the cingulate gyrus (arrows $=$ lesion border). (D) Subpial lesion at high magnification. In comparison, leucocortical (E) and intracortical (F) lesions are smaller and often perivenular in distribution. Broken lines = GM/WM boundary. GM, grey matter; PLP, proteolipid protein; WM, white matter. Scale bars $500 \mu \mathrm{m}$.
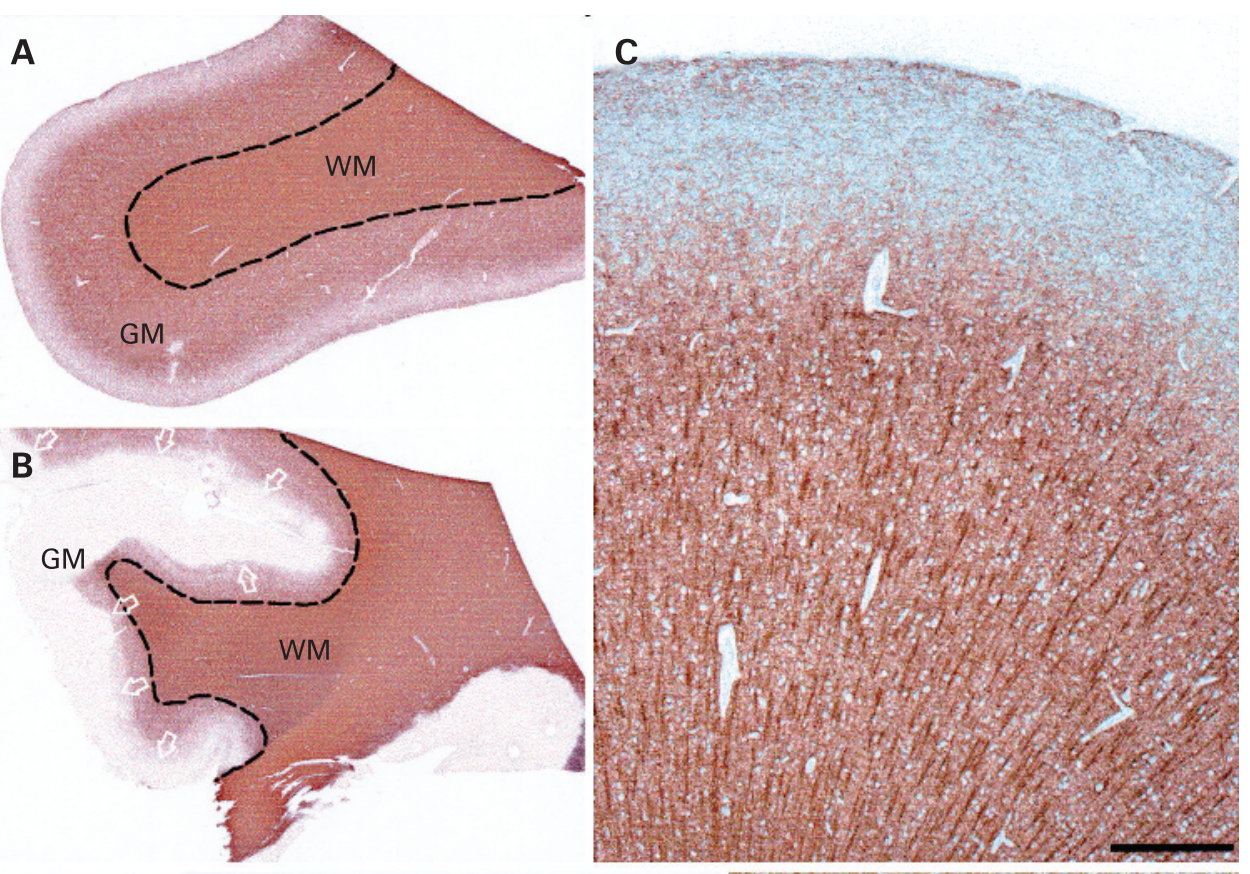

D

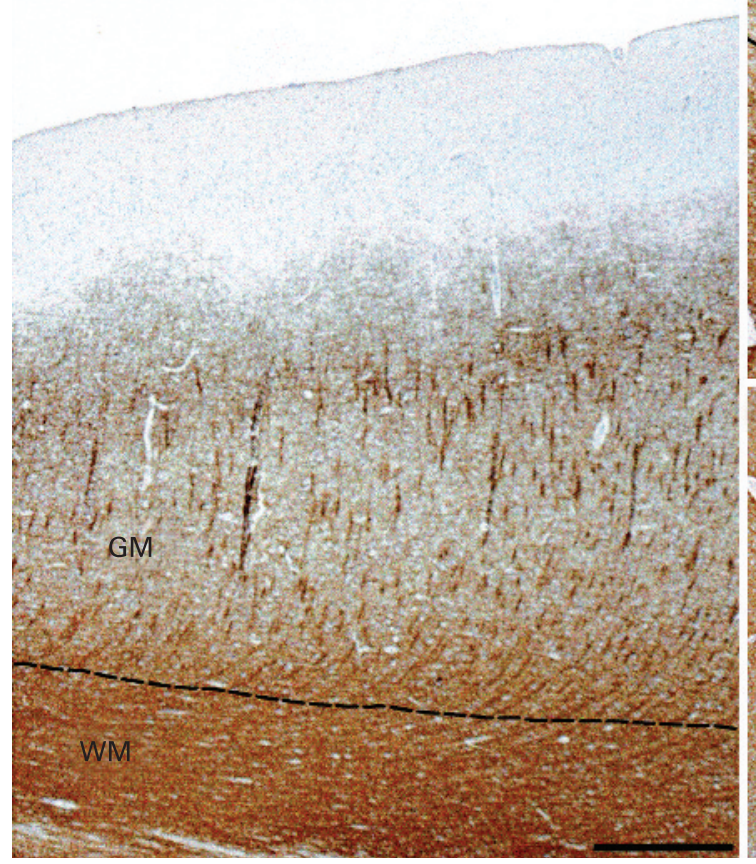



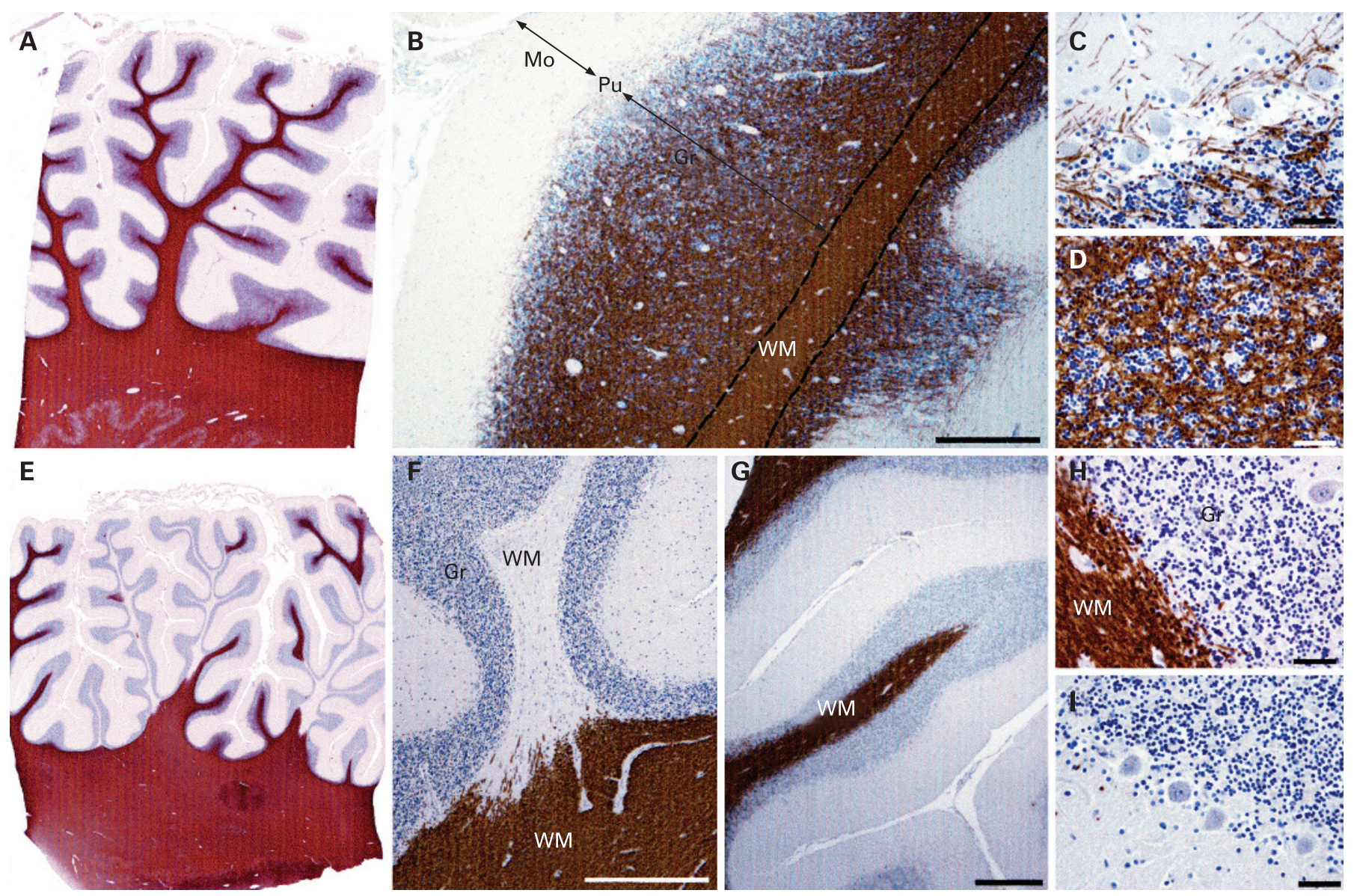

Figure 2 PLP immunostaining of the cerebellum. (A-D) Control; in comparison with the WM, there is a paucity of myelin within the Gr and Pu layers, while the outermost Mo layer is devoid of myelin. (C, D) Pu (C) and $\mathrm{Gr}(\mathrm{D})$ layers at higher magnification. (E-I) Multiple sclerosis case. (E, F) Mixed GM/ WM lesions. (G, H) A proportion of cerebellar plaques are characterised by complete myelin loss in the cortex, with sparing of the subcortical WM. (I) Demyelination in the Gr layer is invariably accompanied by demyelination in the Pu layer and vice versa. GM, grey matter; Gr, granular cell; Mo, molecular; PLP, proteolipid protein; Pu, Purkinje; WM, white matter.

assessed using Spearman's ranked test. Multiple regression analyses were used to examine the influence of age, anatomical location and disease duration on $\mathrm{P}_{\mathrm{GMd}}$ and $\mathrm{P}_{\mathrm{WMd}}$ (Stata V.9; StataCorp, Texas, USA).

\section{RESULTS}

\section{Lesion counts and morphology}

The frequency of GM and WM plaques in each CNS region is shown in table 1. Within the cerebral cortex, thalamus and cerebellum, the majority of GM plaques were restricted to the GM and did not impinge on the WM $(95.8 \%, 87.5 \%$ and $50.6 \%$, respectively). In contrast, only $16.7 \%$ of spinal cord GM plaques involved the GM exclusively. The majority of lesions in the cerebral cortex were subpial in location ( $72 \%$ by number, $96 \%$ by area). Plaque morphology is illustrated in fig 1 (cerebral cortex), fig 2 (cerebellar cortex) and fig 3 (thalamus and spinal cord). We observed evidence of ongoing demyelination (ie, macrophages containing PLP inclusions) in only one case (one subcortical WM lesion and four thalamic lesions-three GM lesions, one mixed GM/WM lesion).

\section{Demyelination is more extensive in the GM than the WM}

Overall, $\mathrm{P}_{\mathrm{GMd}}$ (mean 28.8\%) was significantly greater than $\mathrm{P}_{\mathrm{WMd}} \quad(15.6 \%) \quad(\mathrm{p}<0.001) . \quad \mathrm{P}_{\mathrm{GMd}}$ correlated with $\mathrm{P}_{\mathrm{WMd}}$ $(\mathrm{r}=0.7621, \mathrm{p}<0.001)$.
$\mathrm{P}_{\mathrm{GMd}}$ was greater than $\mathrm{P}_{\mathrm{WMd}}$ in the motor cortex $(\mathrm{p}=0.0128)$, cerebellum $(p=0.0055)$ and spinal cord (cervical, $p=0.0464$; thoracic, $p=0.0082$; lumbar, $p=0.0693$ ) (fig 4). The difference was not significant in the cingulate $(p=0.1185)$ or thalamus $(p=0.3885)$.

\section{Regional differences in the extent of demyelination}

To examine for regional differences in the extent of GM demyelination, $\mathrm{P}_{\mathrm{GMd}}$ was regressed on anatomical location controlling for age and disease duration. In comparison with the cervical cord, $\mathrm{P}_{\mathrm{GMd}}$ was significantly reduced in the motor cortex (coefficient of regression (coeff) $=-0.4703, p<0.001$-ie, $\mathrm{P}_{\mathrm{GMd}}$ was, on average, $47 \%$ greater in the cervical cord than in the motor cortex), the cingulate (coeff $=-0.3220, p=0.001$ ) and the thalamus (coeff $=-0.4449, p<0.001)$. $P_{G M d}$ was not significantly different in the cerebellum (coeff $=-0.0217$, $p=0.875)$, thoracic cord (coeff $=0.0984, p=0.416$ ) or lumbar cord (coeff $=-0.0829, p=0.538$ ) in comparison with the cervical cord. $\mathrm{P}_{\mathrm{GMd}}$ was greater in the cingulate than in the motor cortex $(p=0.013)$.

In comparison with the lumbar cord, $\mathrm{P}_{\mathrm{WMd}}$ was reduced in the WM adjacent to the motor cortex (coeff $=-0.2975$, $p=0.003$ ), cingulate (coeff $=-0.2499, p=0.014)$, thalamus (coeff $=-0.2548, p=0.021)$ and cerebellum (coeff $=-0.1982$, $\mathrm{p}=0.053$ ) but not the cervical (coeff $=-0.0829, \mathrm{p}=0.538$ ) or thoracic cord (coeff $=0.0984, p=0.416$ ). 
Figure 3 PLP immunostaining of the thalamus (A-D) and spinal cord (E-G). (A) Control showing GM nuclei interspersed with WM. (B-G) Multiple sclerosis cases. (B) Multiple demyelinated plaques, largely within the GM. (C, D) Subependymal periventricular GM plaque. (E) Two mixed GM/WM plaques in the cervical cord, both displaying a complete disregard for the GM/WM boundary. Note the paucity of myelin staining within the SG of the $\mathrm{GM}$, also observed in controls. (F, G) A proportion of spinal plaques maintain a strict respect for the GM/WM boundary. Broken lines $=\mathrm{GM} / \mathrm{WM}$ boundary. GM, grey matter; PLP, proteolipid protein; SG, substantia gelatinosa; WM, white matter. Scale bars $500 \mu \mathrm{m}$.
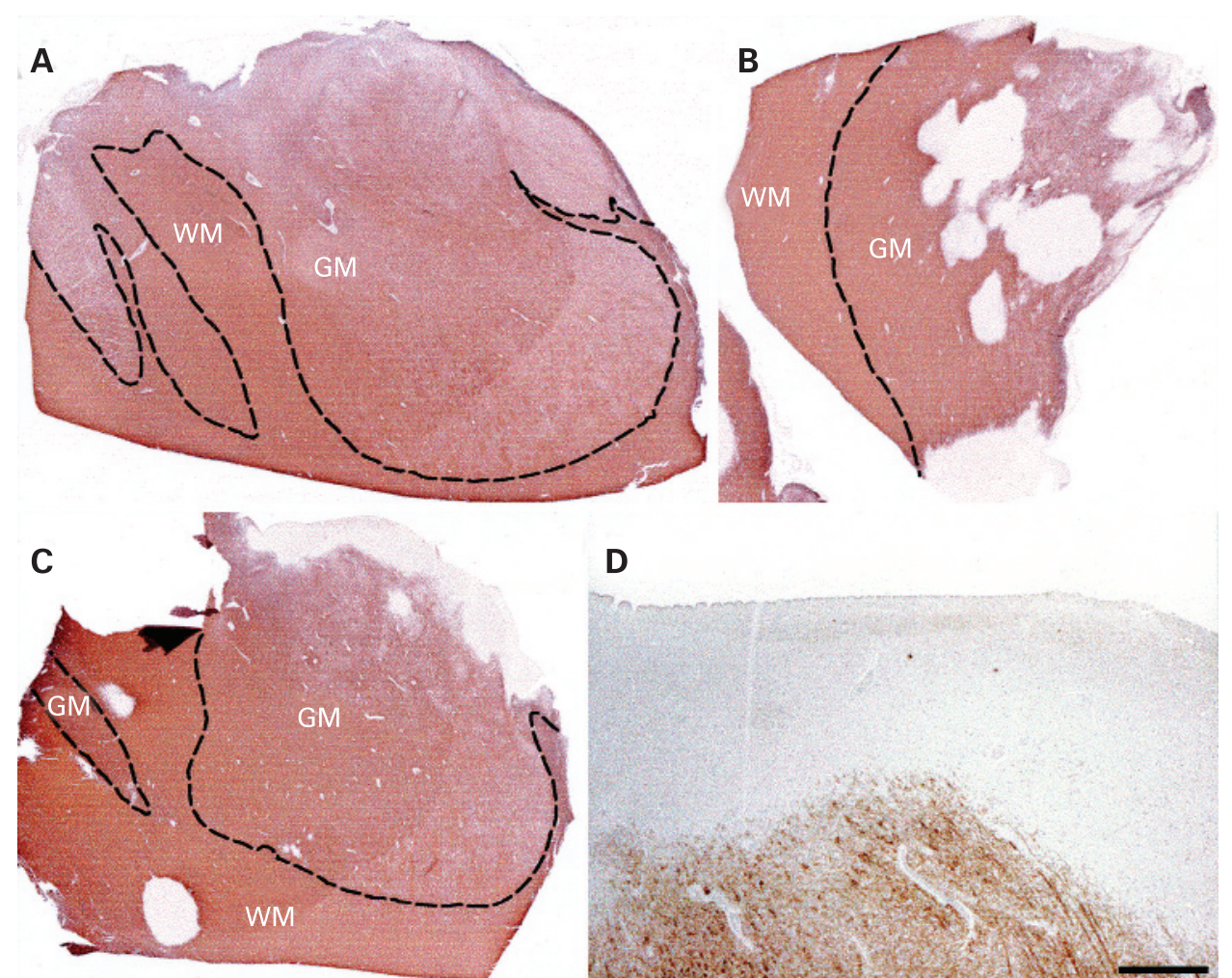

D
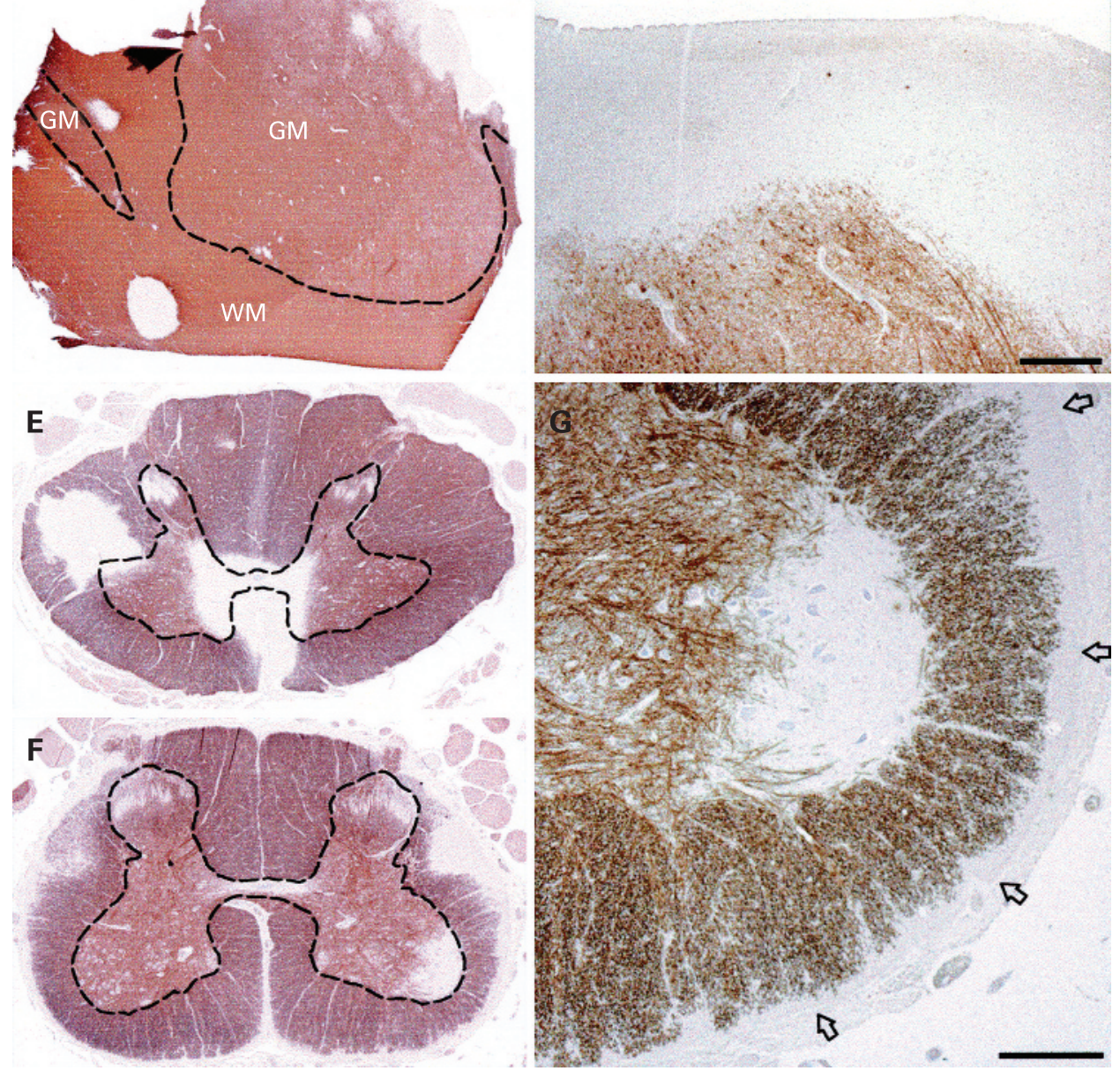

The correlation between $\mathrm{P}_{\mathrm{GMd}}$ and $\mathrm{P}_{\mathrm{WMd}}$ varied between sites, being greatest in the spinal cord (eg, thoracic cord; $r=0.908, p<0.001)$ and thalamus $(r=0.569, p=0.042)$. The two variables did not correlate in the cerebral cortex (eg, cingulate; $r=0.161, p=0.6)$ and cerebellum $(r=0.534$, $\mathrm{p}=0.060) . \mathrm{P}_{\mathrm{GMd}}$ in the cingulate correlated with $\mathrm{P}_{\mathrm{GMd}}$ in the cerebellar cortex $(r=0.745, p=0.003)$, but not in the spinal cord (eg, thoracic cord; $r=0.407, p=0.214$ ).

\section{Influence of age, sex and disease duration on extent of demyelination}

Age (coefficient of regression (coeff) $=-0.0134, p=0.004$ ) appeared to have a significant influence on $\mathrm{P}_{\mathrm{GMd}}$, with younger patients having greater GM demyelination. Disease duration did not appear to influence $\mathrm{P}_{\mathrm{GMd}}(\operatorname{coeff}=0.0091, \mathrm{p}=0.179)$.
Both age (coeff $=-0.0100, p=0.003)$ and disease duration (coeff $=0.0092, p=0.049$ ) appeared to have a significant influence on $\mathrm{P}_{\mathrm{WMd}}$ with more extensive demyelination being observed in younger patients and those with longer disease durations.

\section{DISCUSSION}

Numerous studies have examined the distribution of MS plaques within the CNS using histochemical myelin stains. ${ }^{23}$ This work has suggested that while demyelination occurs in both WM and GM structures, MS is predominantly a WM disease. However, such studies are likely to have substantially underestimated the extent of GM involvement. ${ }^{467}$ In light of this, it is essential that the extent and pattern of demyelination in $\mathrm{MS}$ is re-evaluated using more sensitive methods of lesion 


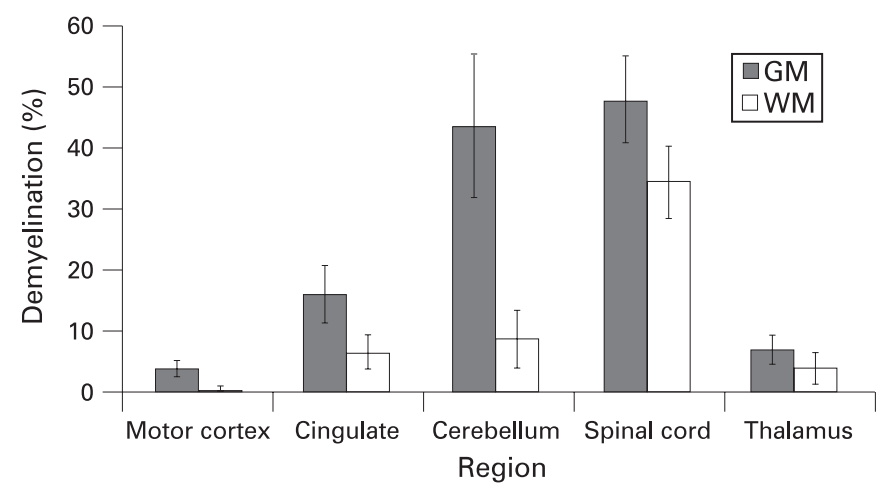

Figure 4 Proportion of grey matter (GM) that is demyelinated and the proportion of white matter (WM) that is demyelinated at the different sites of the CNS. Values represent mean (SE). Spinal cord values represent the pooled results from the three cord levels.

detection. Therefore, we have used myelin protein immunohistochemistry - the "gold standard" technique for detecting GM demyelination - to evaluate demyelination in the cerebral cortex, cerebellum, thalamus and spinal cord.

While several studies have compared the frequency of MS plaques within different brain regions, few have included tissue from the spinal cord. ${ }^{8}$ We observed a striking preponderance for WM demyelination in the cord in comparison with the other WM structures examined. The extent of GM demyelination is also much greater in the spinal cord than in the cerebral cortex and deep GM structures. However, in contrast with the situation in the WM, the extent of GM demyelination in the cerebellum is comparable to that in the cord. We note that plaques which cause particularly disabling symptoms are likely to be overrepresented in an autopsy series; such sites include the brainstem and spinal cord. Therefore, while anatomical factors may indeed predispose to plaques in these areas, this may be exaggerated by sampling bias.

$\mathrm{P}_{\mathrm{GMd}}$ was greater than $\mathrm{P}_{\mathrm{WMd}}$ in all of the regions studied (fig 4); these differences were significant in the motor cortex, cerebellum and spinal cord. We cannot conclusively state that demyelination is more extensive in the GM than in the WM as several areas have not been sampled, notably the periventricular tissue, brainstem and optic nerves. However, we have included material from the spinal cord, subcortical WM, corpus callosum (present on the cingulate sections) and the cerebellum, all of which are considered predilection sites for WM plaques. ${ }^{1}$

\section{Demyelination in the cerebral and cerebellar cortex}

In keeping with previous studies, the majority of cerebral cortical lesions were subpial in location. ${ }^{45}$ These often involved extensive lengths of the cortical ribbon. Consistent with Kutzelnigg et al, we found no correlation between $\mathrm{P}_{\mathrm{GMd}}$ and $\mathrm{P}_{\mathrm{WMd}}$ in the cerebral cortex, suggesting that cortical demyelination, particularly subpial demyelination, occurs by an independent mechanism to WM demyelination. ${ }^{5}$ Subpial lesions do not appear to occur in a perivenous distribution; they frequently cover extensive areas, extending from the surface of the brain into the cerebral cortex. ${ }^{4}$ It has been suggested that subpial demyelination is mediated by a "myelinotoxic factor", generated by inflammatory cells in the meninges or the CSF, diffusing inward from the surface of the brain. ${ }^{49}$ Kutzelnigg et al found subpial demyelination to be most pronounced within deep invaginations of the cortex, including the cingulate, possibly reflecting regional differences in CSF flow, with extensive demyelination occurring in areas of CSF stasis. ${ }^{9}$

Consistent with a recent study, we have demonstrated that the cerebellar cortex is a predilection site for demyelination, with $\mathrm{P}_{\mathrm{GMd}}$ exceeding $70 \%$ in six of the 13 cases examined in the current study. ${ }^{10}$ The cerebellum also showed the greatest difference between $\mathrm{P}_{\mathrm{GMd}}$ (mean 43.7\%) and $\mathrm{P}_{\mathrm{WMd}}$ (mean $8.7 \%$ ). Like the subpial plaques of the cerebral cortex, subpial cerebellar lesions are extensive, typically involving multiple folia. $\mathrm{P}_{\mathrm{GMd}}$ in the cingulate correlates with $\mathrm{P}_{\mathrm{GMd}}$ in the cerebellum, but not with $\mathrm{P}_{\mathrm{GMd}}$ in the spinal cord, further suggesting that subpial demyelination occurs by a different mechanism to demyelination at other sites.

Both subpial cerebellar lesions and the so-called type IV plaques of the cerebral cortex involve the full thickness of the cortex, without impinging on the subcortical WM. ${ }^{4} \mathrm{~A}$ proportion of spinal cord plaques also show strict respect for the GM/ WM boundary, suggesting a common pathogenic mechanism. We have previously suggested that this pattern of plaque morphology may reflect a relative failure of remyelination in the GM, giving rise to extensive areas of demyelinated GM with apparent sparing of the adjacent WM. ${ }^{6}$ Alternatively, differences in the extent of demyelination between WM and GM structures may stem from antigenic differences between GM myelin and WM myelin or differences in the handling of interstitial fluid between WM and GM.

The hypothesis that demyelination is mediated by a myelinotoxic factor in the interstitial fluid is an attractive one. Interstitial fluid in GM drains preferentially along perivascular (Virchow Robin) channels to the CSF, while interstitial fluid in WM also spreads through the extracellular spaces between nerve fibres; in the WM of the brain this fluid predominantly drains into the ventricles. ${ }^{11}$ There is some evidence that interstitial fluid is cleared more efficiently from WM than GM. ${ }^{11}$ GM/WM differences in interstitial fluid drainage may thereby result in areas of GM demyelination with sparing of the adjacent WM. Furthermore, a myelinotoxic substance originating in the interstitial fluid could potentially circulate within the Virchow Robin space (resulting in perivenular demyelination) and the CSF (mediating subpial and periventricular demyelination). ${ }^{12}$ We acknowledge that the majority of plaques in our postmortem series are likely to be longstanding. Because of a paucity of actively demyelinating lesions, we have not been able to adequately assess plaque morphology in the early stages of lesion development.

\section{Spinal cord and thalamic demyelination}

We have previously examined GM demyelination in the spinal cord in detail. ${ }^{6}$ In the current study, we have confirmed the finding of substantial GM involvement throughout the cord. We noted that despite our speculations that subpial demyelination is mediated by a factor related to the CSF, GM demyelination is even more extensive in the spinal cord where the GM is not bathed in CSF. Animal studies indicate marked differences between the GM of the brain and spinal cord in terms of their response to injury. Following injection of proinflammatory cytokines, leucocyte infiltration and bloodbrain barrier breakdown is more pronounced in the spinal cord GM in comparison with the striatum. ${ }^{13}{ }^{14}$ Similar differences between sites may result in regional differences in the extent of GM demyelination.

Consistent with previous work, we detected a modest degree of demyelination in the thalamus. ${ }^{7}$ The plaque borders within the deep GM structures did not appear to respect the GM/WM 
boundary. While the ependymal surface was not identifiable on all of the sections, subependymal GM plaques were present in a number of cases, sometimes extending over a relatively large area (fig 3C). While the majority of periventricular WM plaques demonstrate a perivenular distribution, a smaller number appear to arise from the ependymal surface of the ventricle itself, suggesting that inflammatory cells or a demyelinating factor within the ventricular system may be responsible for the formation of a proportion of plaques. ${ }^{15}$ Further work is required to examine the relationship between periventricular GM plaques and subependymal veins and to compare the extent of periventricular demyelination between GM and WM structures.

\section{Influence of disease duration and MS subtype}

In agreement with previous work, we found no correlation between disease duration and the extent of GM demyelination. ${ }^{5}{ }^{6}$ However, disease duration was less than 10 years in only one subject; the lesion counts observed therefore reflect years of cumulative pathology. Studies on patients with shorter disease durations are required to determine whether GM demyelination is an early or late event or indeed whether it occurs consequent to WM injury.

Kutzelnigg et al have suggested cortical demyelination may be a pathological correlate of disease progression. ${ }^{5}$ Because of a paucity of PPMS and RRMS cases, we have been unable to investigate the influence of MS subtype on the extent of demyelination. However, in the single RRMS case, $18.7 \%$ of the cingulate was demyelinated, suggesting extensive cortical demyelination is not exclusively a feature of progressive disease. Interestingly no cortical plaques were detected in the two PPMS cases, although we have examined relatively small areas of cortex.

\section{CONCLUSION}

We demonstrated substantial variation in the extent of both GM and WM demyelination between different regions of the CNS. Of the areas examined, GM demyelination was most extensive in the spinal cord and cerebellum, while WM demyelination was most prominent in the spinal cord. Demyelination was greater in the GM than in the WM at each anatomical site. Further work is required to better characterise these GM plaques-for example, examining inflammatory cell infiltration, blood-brain barrier breakdown, gliosis and remyelination -in order to understand the factors that influence lesion topography and the apparent preponderance for GM demyelination.

Acknowledgements: Abhi Vora of the UK Multiple Sclerosis Brain Bank provided the autopsy material.

Funding: The study was supported by funding from the Multiple Sclerosis Society of Great Britain and Northern Ireland (grant No 801/03, NE).

Competing interests: None.

Ethics approval: The local research ethics committee approved the study.

\section{REFERENCES}

1. Lassmann H. Pathology of multiple sclerosis. In: Compston A, Ebers G, McDonald I, et al, eds. McAlpines multiple sclerosis, 3rd Edn. London: Churchill Livingstone, 1998:323-58.

2. Fog T. Topographic distribution of plaques in the spinal cord in multiple sclerosis. Arch Neurol Psychiatry 1950;63:382-414.

3. Brownell B, Hughes JT. The distribution of plaques in the cerebrum in multiple sclerosis. J Neurol Neurosurg Psychiatry 1962;25:315-20.

4. Bo L, Vedeler CA, Nyland HI, et al. Subpial demyelination in the cerebral cortex of multiple sclerosis patients. J Neuropathol Exp Neurol 2003;62:723-32.

5. Kutzelnigg A, Lucchinetti CF, Stadelmann C, et al. Cortical demyelination and diffuse white matter injury in multiple sclerosis. Brain Nov 2005;128:2705-12.

6. Gilmore CP, Bo L, Owens T, et al. Spinal cord gray matter demyelination in multiple sclerosis - a novel pattern of residual plaque morphology. Brain Pathol 2006;16:202-8.

7. Vercellino M, Plano F, Votta B, et al. Grey matter pathology in multiple sclerosis. J Neuropathol Exp Neurol 2005;64:1101-7.

8. Raine CS. The neuropathology of multiple sclerosis. In: Raine CS, McFarland HF, Tourtellotte WM, eds. Multiple sclerosis. London: Chapman and Hall Medical, 1997:151-72.

9. Kutzelnigg A, Lassmann $\mathrm{H}$. Cortical demyelination in multiple sclerosis: A substrate for cognitive deficits? J Neurol Sci 15 2006;245:123-6.

10. Kutzelnigg A, Faber-Rod JC, Bauer J, et al. Widespread demyelination in the cerebellar cortex in multiple sclerosis. Brain Pathol 2007:17:38-44.

11. Weller RO, Kida S, Zhang ET. Pathways of fluid drainage from the brainmorphological aspects and immunological significance in rat and man. Brain Pathol 1992:2:277-84.

12. Gay FW. Early cellular events in multiple sclerosis. Intimations of an extrinsic myelinolytic antigen. Clin Neurol Neurosurg 2006;108:234-40.

13. Schnell L, Fearn S, Klassen $\mathrm{H}$, et al. Acute inflammatory responses to mechanica lesions in the CNS: differences between brain and spinal cord. Eur J Neurosci 1999;11:3648-58.

14. Schnell L, Fearn S, Schwab ME, et al. Cytokine-induced acute inflammation in the brain and spinal cord. J Neuropathol Exp Neurol 1999;58:245-54.

15. Adams CW, Abdulla YH, Torres EM, et al. Periventricular lesions in multiple sclerosis: their perivenous origin and relationship to granular ependymitis. Neuropathol Appl Neurobiol 1987;13:141-52. 


\section{JNNP}

\section{Regional variations in the extent and pattern of grey matter demyelination in multiple sclerosis: a comparison between the cerebral cortex, cerebellar cortex, deep grey matter nuclei and the spinal cord}

C P Gilmore, I Donaldson, L Bö, et al.

J Neurol Neurosurg Psychiatry 2009 80: 182-187 originally published online October 1, 2008

doi: 10.1136/jnnp.2008.148767

Updated information and services can be found at:

http://jnnp.bmj.com/content/80/2/182.full.html

\section{These include:}

References This article cites 11 articles, 2 of which can be accessed free at: http://jnnp.bmj.com/content/80/2/182.full.html\#ref-list-1

Article cited in:

http://jnnp.bmj.com/content/80/2/182.full.html\#related-urls

Email alerting Receive free email alerts when new articles cite this article. Sign up in service the box at the top right corner of the online article.

Topic Articles on similar topics can be found in the following collections Collections

Immunology (including allergy) (45820 articles)

Brain stem / cerebellum (2141 articles)

Multiple sclerosis (1662 articles)

Notes

To request permissions go to:

http://group.bmj.com/group/rights-licensing/permissions

To order reprints go to:

http://journals.bmj.com/cgi/reprintform

To subscribe to BMJ go to:

http://group.bmj.com/subscribe/ 